\title{
An empirical model of the auroral oval derived from CHAMP field-aligned current signatures - Part 2
}

\author{
C. Xiong ${ }^{1,2}$ and H. Lühr ${ }^{1}$ \\ ${ }^{1}$ Helmholtz Centre Potsdam, GFZ German Research Centre for Geosciences, Telegrafenberg, 14473 Potsdam, Germany \\ ${ }^{2}$ Department of Space Physics, College of Electronic Information, Wuhan University, 430079 Wuhan, China \\ Correspondence to: C. Xiong (bear@gfz-potsdam.de)
}

Received: 5 February 2014 - Revised: 23 April 2014 - Accepted: 24 April 2014 - Published: 4 June 2014

\begin{abstract}
In this paper we introduce a new model for the location of the auroral oval. The auroral boundaries are derived from small- and medium-scale field-aligned current (FAC) based on the high-resolution CHAMP (CHAllenging Minisatellite Payload) magnetic field observations during the years 2000-2010. The basic shape of the auroral oval is controlled by the dayside merging electric field, $E_{\mathrm{m}}$, and can be fitted well by ellipses at all levels of activity. All five ellipse parameters show a dependence on $E_{\mathrm{m}}$ which can be described by quadratic functions. Optimal delay times for the merging electric field at the bow shock are 30 and $15 \mathrm{~min}$ for the equatorward and poleward boundaries, respectively. A comparison between our model and the British Antarctic Survey (BAS) auroral model derived from IMAGE (Imager for Magnetopause-to-Aurora Global Exploration) optical observations has been performed. There is good agreement between the two models regarding both boundaries, and the differences show a Gaussian distribution with a width of $\pm 2^{\circ}$ in latitude. The difference of the equatorward boundary shows a local-time dependence, which is $1^{\circ}$ in latitude poleward in the morning sector and $1^{\circ}$ equatorward in the afternoon sector of the BAS model. We think the difference between the two models is caused by the appearance of auroral forms in connection with upward FACs. All information required for applying our auroral oval model (CH-Aurora-2014) is provided.
\end{abstract}

Keywords. Ionosphere (auroral ionosphere) - magnetospheric physics (current systems; magnetosphereionosphere interactions)

\section{Introduction}

The auroral oval is the region in the ionosphere receiving the main part of the magnetospheric particle precipitation. It usually appears as luminous bands surrounding both geomagnetic poles at ionospheric altitudes (Feldstein, 1963; Khorosheva, 1967; Frey, 2007). One important feature of the auroral oval pattern is that it approximately forms the boundary of the polar cap, inside which the magnetic field lines are open and merged with interplanetary magnetic field (IMF) lines. The auroral oval is quite dynamic and generally related to the prevailing geomagnetic activity. The exact location of the equatorward oval boundary depends both on the energy of the precipitating particles and the magnetospheric electric and magnetic fields (Kauristie et al., 1999), while the size of the polar cap depends on the amount of magnetic field energy stored in the tail lobes. The locations of the auroral boundaries are important parameters for estimating the energy flows in the solar-wind-magnetosphere-ionosphere system (Baker et al., 1997). Therefore, monitoring the whole auroral oval on a quasi-instantaneous basis is of great interest for the study of magnetospheric dynamics.

Based on DMSP (Defense Meteorological Satellite Program) auroral photographs, there have already been some early model attempts for predicting the location of the auroral oval (Feldstein and Starkov, 1970; Holzworth and Meng, 1975; Hardy et al., 2008) and the global distribution of the electrons and ions streaming into the ionosphere (Hardy et al., 1985). However, these observations did not cover all the magnetic local times (MLT) well, in particular in the Southern Hemisphere. A much improved model of the auroral oval was derived from optical observations of the IMAGE (Imager for Magnetopause-to-Aurora Global Exploration) 
satellite. This spacecraft was able to provide pictures of the whole oval every 2-3 min. Based on these data, Boakes et al. (2008) and Longden et al. (2010) developed techniques for an automatic detection of the auroral boundaries. A corresponding data set containing the boundary locations in the Northern Hemisphere for the years May 2000October 2002 is available at the British Antarctic Survey (BAS) server. Using 10 years of continuous magnetic field observations from CHAMP (CHAllenging Minisatellite Payload), Xiong et al. (2014) developed an alternative method for detecting the auroral boundaries from field-aligned current signatures. In a statistical analysis they demonstrated the reliability of their approach. In this study we will make use of this boundary detection approach to develop an empirical model for the auroral oval. Different from the BAS model we cover both hemispheres and provide oval positions for the whole solar cycle from 2000 to 2010.

In the sections to follow we first briefly repeat the detection approach described by Xiong et al. (2014). The main part, Sect. 3, is concerned with the design of suitable control parameters determining the shape of the oval boundaries. It is followed by a detailed description of the processing steps. A systematic validation of our model versus others, in particular the BAS model, is presented in Sect. 4. Important features of the model are summarized in Sect. 5 .

\section{Data and processing approach}

The CHAMP satellite was launched on 15 July 2000 into a circular, near-polar orbit (inclination: $87.3^{\circ}$ ) with an initial altitude of $456 \mathrm{~km}$. By the end of the mission, 17 September 2010, the orbit had decayed to $250 \mathrm{~km}$. For this study we make use of the fluxgate magnetometer (FGM) on board CHAMP that provides magnetic vector field readings at a rate of $50 \mathrm{~Hz}$ and a resolution of $0.1 \mathrm{nT}$. The data were calibrated routinely with respect to the onboard absolute scalar Overhauser magnetometer (OVM) and provided as Level-2 readings $(1 \mathrm{~Hz})$.

For our boundary detections we make use of the two transverse components in a mean-field-aligned (MFA) coordinate system, which is suitable for calculating field-aligned currents (FAC). The mid-point of a significant increase of FAC intensity has been used to determine the auroral boundaries. Details of the MFA coordinates and the applied FAC determination are given in Xiong et al. (2014). The FAC intensities calculated by our approach reflect mainly the small- and medium-scale $(<150 \mathrm{~km})$ field-aligned currents. The advantage of favoring the smaller scale FACs is that boundaries appear sharper. Further, an automatic approach for detecting the boundaries of the auroral oval based on the intensity of FACs has been developed and applied by Xiong et al. (2014). Here we make use of the derived boundary locations from the whole CHAMP mission.

\section{The auroral oval model}

\subsection{The controlling parameter for the model}

In our previous study (Xiong et al., 2014), 10 years of CHAMP observations from August 2000 to August 2010 have been used for studying the characteristics of the auroral oval. We have tested the functional relations between the latitudes of the auroral boundaries and different magnetic activity indices. Best results for a linear dependence were derived for both boundaries when using the merging electric field. The other indices, like auroral electrojet (AE) index and Dst, also provide good linear relations but with some caveats. Therefore, we choose the merging electric field derived from solar wind data for characterizing the expansion of the auroral oval and for parameterizing the model in this study.

The merging electric field we used is the new coupling function as defined by Newell et al. (2007):

$E_{\mathrm{m}}^{\prime}=V_{\mathrm{sw}}^{\frac{4}{3}}\left(\sqrt{B_{y}{ }^{2}+B_{z}{ }^{2}}\right)^{\frac{2}{3}} \sin ^{\frac{8}{3}}\left(\frac{\theta}{2}\right)$,

where $V_{\mathrm{sw}}$ denotes the solar wind velocity, $B_{y}$ and $B_{z}$ denote the $y$ and $z$ components of the interplanetary magnetic field (IMF) in geocentric solar magnetospheric (GSM) coordinates, and $\theta$ is the clock angle of the $\operatorname{IMF}\left(\tan (\theta)=\frac{\left|B_{y}\right|}{B_{3}}\right)$. To make the numerical values comparable with the merging electric field (in $\mathrm{mV} \mathrm{m}^{-1}$ ) defined by Kan and Lee (1979), we use $V_{\mathrm{sw}}$ in units of $\mathrm{km} \mathrm{s}^{-1}$ and $B_{y}$ and $B_{z}$ in nT for calculating the $E_{\mathrm{m}}^{\prime}$ in Eq. (1) and then divide the value by a factor of 1000 .

An important characteristic of the magnetosphereionosphere system that has to be taken into account (Werner and Pröss, 1997; Liu et al., 2010) is its "memory effect" with respect to solar wind input changes . The time-integrated $E_{\mathrm{m}}$ can be defined as

$E_{\mathrm{m}}(t, \tau)=\frac{\int_{t_{1}}^{t} E_{\mathrm{m}}^{\prime}\left(t^{\prime}\right) e^{\left(t^{\prime}-t\right) / \tau} \mathrm{d} t^{\prime}}{\int_{t_{1}}^{t} e^{\left(t^{\prime}-t\right) / \tau} \mathrm{d} t^{\prime}}$,

where $E_{\mathrm{m}}^{\prime}$ is treated as a continuous function of time $t^{\prime}, t_{1}$ is chosen $3 \mathrm{~h}$ before the actual epoch, and $\tau$ is the e-folding time of the weighting function in the integrands. In this study $\tau=$ $0.5 \mathrm{~h}$ is found to be the most suitable for our model. The values for $t_{1}$ and $\tau$ were determined experimentally, providing the best linear relation between the retarded $E_{\mathrm{m}}$ and latitudes of the boundaries.

\subsection{Ellipse fitting to the auroral oval}

In this study we use the approach presented in Xiong et al. (2014) to determine the auroral boundaries from the CHAMP FAC signatures. In order to describe the auroral boundaries by analytical functions all the detections are divided into bins of different activity levels as defined by $E_{\mathrm{m}}$. To make sure that the numbers of samples in each $E_{\mathrm{m}}$ bin is large enough, 

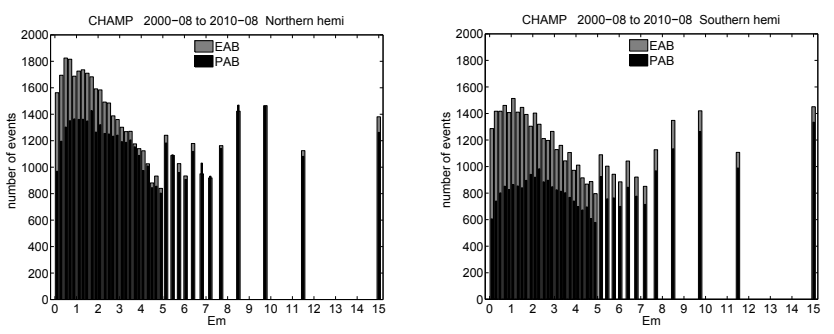

Figure 1. The number of events of the auroral oval boundaries in each $E_{\mathrm{m}}$ bin for Northern (left) and Southern (right) Hemispheres. "EAB" and "PAB" represent the equatorward and poleward auroral boundary, respectively.

the sizes of $E_{\mathrm{m}}$ bins are different for different activity levels. The $E_{\mathrm{m}}$ range and center value of each bin is listed in Table 1. Figure 1 presents the distribution of the number of events separately for both boundaries and hemispheres. "EAB" and "PAB" represent the equatorward and poleward auroral boundary, respectively. The sizes of the bins have been chosen such that the number of events is more than 1000 and 800 for EAB and PAB, respectively. These large numbers of detections will guarantee a sufficient number of entries at all MLTs and activity bins.

The boundary detections of each $E_{\mathrm{m}}$ bin are first divided into 24 MLT bins ( $2 \mathrm{~h}$ wide overlapping bins). From these values the central latitudes of the boundaries are determined for the different MLTs. The latitude distributions of the boundary detection occurrence rates for the bin around midnight are presented in Fig. 2 (left column) for four examples of $E_{\mathrm{m}}$ levels. From top to bottom the values of $E_{\mathrm{m}}$ cover the ranges: $0.2-0.4,1.8-2.0,5.0-5.3$, and 9.0-10.5, respectively. All the latitude profiles of occurrence rates resemble Gaussian normal distributions quite well, and the latitudes at peak values can be taken as representative of the $E_{\mathrm{m}}$ activity level. It can be clearly seen that with increasing $E_{\mathrm{m}}$ the center latitude of the equatorward boundary appears at 67 , 65,62 , and $59^{\circ}$ in apex latitude, while the poleward boundary stays between 72 and $74^{\circ}$ in apex latitude. For the actual determination of the mean latitude in each MLT bin, only the detections within the latitude range where the occurrence rate is larger than $5 \%$ are used. This is done to eliminate the influence of individual outliers. The derived mean latitudes of the detections are presented in Fig. 2 (right column) for every MLT hour as black and red dots for the equatorward and poleward auroral boundaries, respectively.

In a next step we use the 24 hourly mean values of the detections in each $E_{\mathrm{m}}$ bin and fit ellipses to them for the two boundaries. As shown in Fig. 3, five parameters are needed to represent an ellipse. The semi $x$ and semi $_{y}$ are the semiaxis of the ellipse in midnight-noon and dawn-dusk directions; $x_{0}$ and $y_{0}$ are the coordinates of the ellipse center, with a positive value of $x, y$ pointing towards midnight and dawn, respectively; $\varphi_{0}$ is the orientation angle of the ellipse
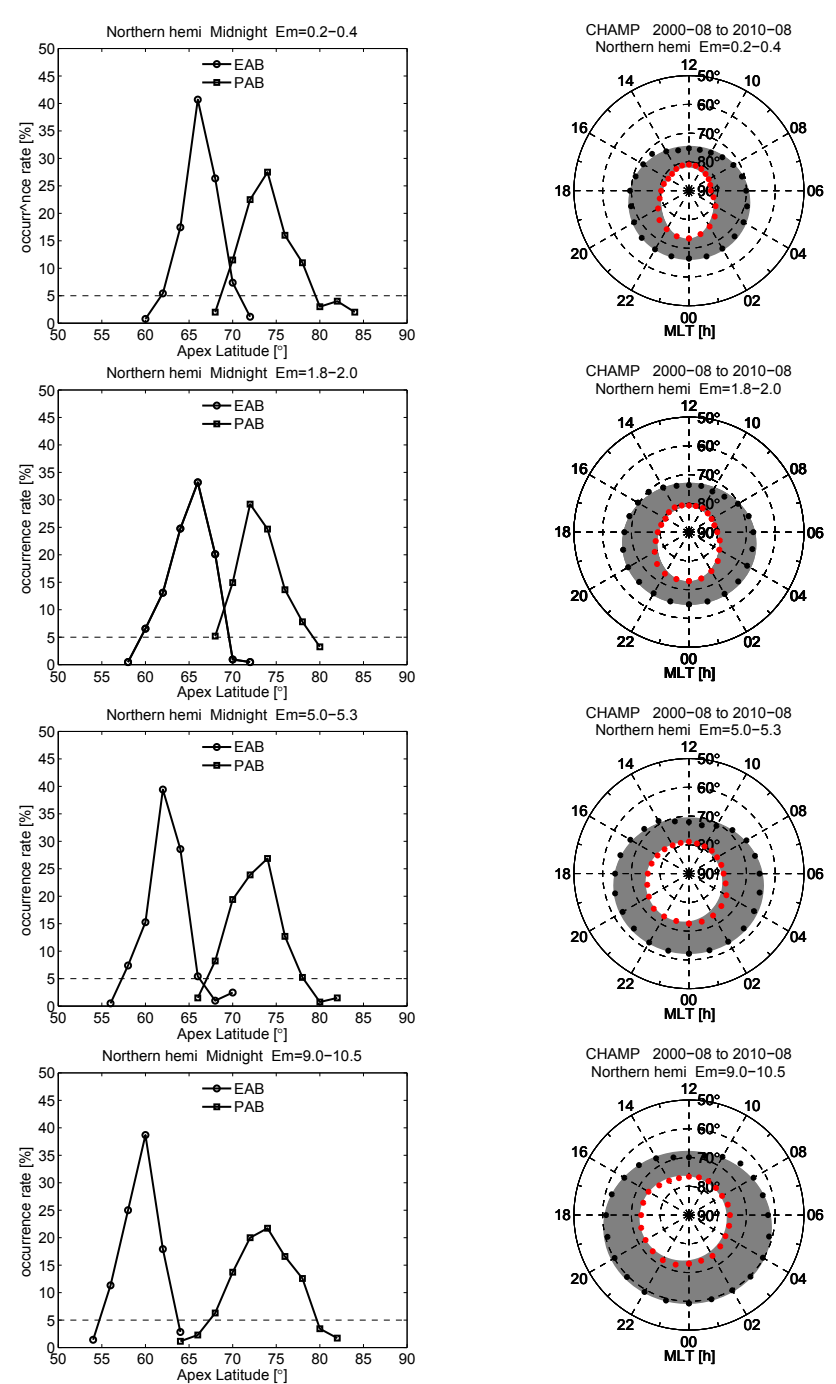

Figure 2. Left: the auroral boundaries' latitude distribution at midnight for different $E_{\mathrm{m}}$ bins. Right: the magnetic latitude and magnetic local-time distribution of equatorward (black) and poleward (red) boundary detections for different $E_{\mathrm{m}}$ bins; solid dots are the mean latitudes of $1 \mathrm{~h}$ bins.

between semi $_{x}$ and the midnight-noon axis, with positive values counting counterclockwise. All the length scales are in degrees of latitude. For most of the cases, the center of the fitted ellipse is not right at the magnetic pole, and the orientation angle of the ellipse is non-zero.

For every angle $\varphi$ measured from the midnight-noon axis we can compute the radius $r_{0}$ from the center of the ellipse:

$$
r_{0}=\frac{\operatorname{semi}_{x} \cdot \operatorname{semi}_{y}}{\sqrt{\left(\operatorname{semi}_{x} \cdot \sin \left(\varphi+\varphi_{0}\right)\right)^{2}+\left(\operatorname{semi}_{y} \cdot \cos \left(\varphi+\varphi_{0}\right)\right)^{2}}} .
$$

When taking the difference between the ellipse center and the magnetic pole into account, the radius $r$ from the magnetic pole, namely the co-latitude of the auroral boundary, can be defined as 
Table 1. The range and mean values of $E_{\mathrm{m}}$ in each bin.

\begin{tabular}{lrrrrrrrr}
\hline Bin No. & Bin size & Central $E_{\mathrm{m}}$ & Bin no. & Bin size & Central $E_{\mathrm{m}}$ & Bin no. & Bin size & Central $E_{\mathrm{m}}$ \\
\hline 1 & 0.2 & 0.1 & 14 & 0.2 & 2.7 & 27 & 0.3 & 5.45 \\
2 & 0.2 & 0.3 & 15 & 0.2 & 2.9 & 28 & 0.3 & 5.75 \\
3 & 0.2 & 0.5 & 16 & 0.2 & 3.1 & 29 & 0.3 & 6.05 \\
4 & 0.2 & 0.7 & 17 & 0.2 & 3.3 & 30 & 0.4 & 6.4 \\
5 & 0.2 & 0.9 & 18 & 0.2 & 3.5 & 31 & 0.4 & 6.8 \\
6 & 0.2 & 1.1 & 19 & 0.2 & 3.7 & 32 & 0.4 & 7.2 \\
7 & 0.2 & 1.3 & 20 & 0.2 & 3.9 & 33 & 0.6 & 7.7 \\
8 & 0.2 & 1.5 & 21 & 0.2 & 4.1 & 34 & 1.0 & 8.5 \\
9 & 0.2 & 1.7 & 22 & 0.2 & 4.3 & 35 & 1.5 & 9.75 \\
10 & 0.2 & 1.9 & 23 & 0.2 & 4.5 & 36 & 2.0 & 11.5 \\
11 & 0.2 & 2.1 & 24 & 0.2 & 4.7 & 37 & $\infty$ & $>12.5$ \\
12 & 0.2 & 2.3 & 25 & 0.2 & 4.9 & & & \\
13 & 0.2 & 2.5 & 26 & 0.3 & 5.15 & & & \\
\hline
\end{tabular}

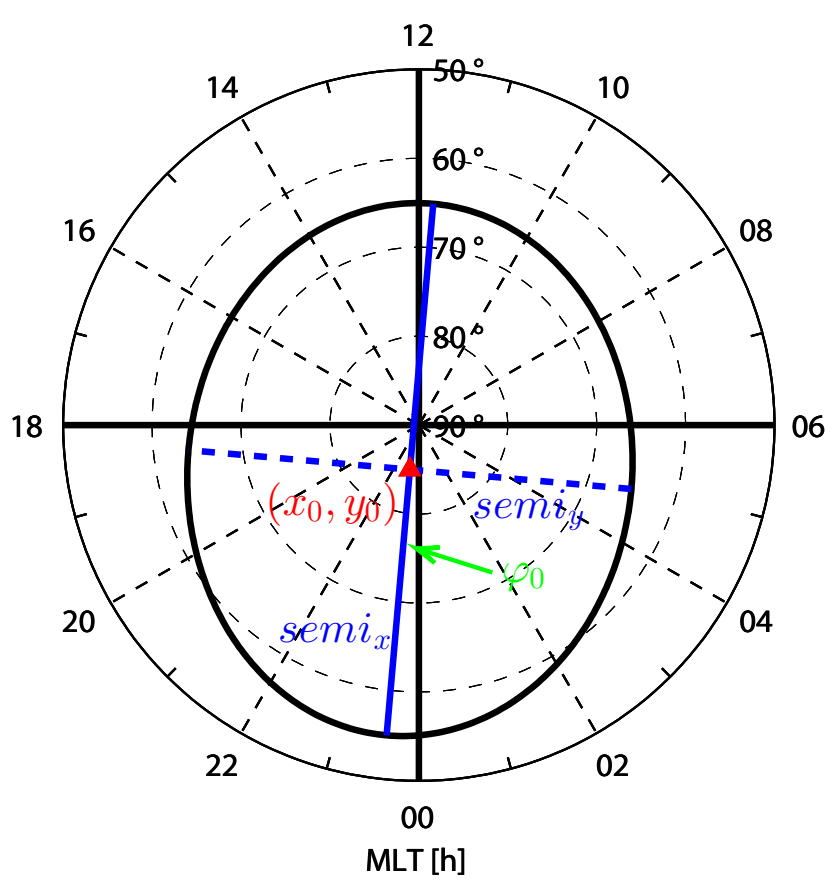

Figure 3. The five parameters of an ellipse: $\operatorname{semi}_{x}$ (blue solid) and semi $_{y}$ (blue dashed) are the semi-axes of the ellipse in midnightnoon and dawn-dusk direction; $x_{0}$ and $y_{0}$ are the coordinates of ellipse center (red triangle), with positive values of $x, y$ pointing towards midnight and dawn, respectively; $\varphi_{0}$ is the orientation angle of the ellipse, with positive values counterclockwise.

$r=\sqrt{\left(r_{0} \cdot \cos \left(\varphi+\varphi_{0}\right)+x_{0}\right)^{2}+\left(r_{0} \cdot \sin \left(\varphi+\varphi_{0}\right)+y_{0}\right)^{2}}$.

In practice, it is more appropriate to have an angular representation in magnetic local time (MLT), $\lambda=2 \pi \cdot \mathrm{MLT} / 24 \mathrm{~h}$. This can be derived as

$\tan (\lambda)=\frac{r \cdot \sin \left(\varphi+\varphi_{0}\right)+y_{0}}{r \cdot \cos \left(\varphi+\varphi_{0}\right)+x_{0}}$.
Due to the offset between the ellipse center and the magnetic pole, the angular value of $\varphi$ is not equal to the local-time angle $\lambda$. Here we use the two-step approximation to minimize the error arising from the difference between $\lambda$ and $\varphi$. For a given MLT, we first use $\lambda$ to replace $\varphi$ in Eqs. (3) and (4), then $\lambda^{\prime}$ is calculated from Eq. (5). The difference between $\lambda$ and $\lambda^{\prime}$ can be defined as

$\Delta \lambda=\lambda^{\prime}-\lambda$

In the second step we can use the desired magnetic local time for quantifying $\varphi$

$\varphi=\lambda+\Delta \lambda$.

Finally, this new ellipse angle will be used as $\varphi$ in Eqs. (3) and (4) to calculate the auroral boundary location.

In order to determine the dependence of the auroral oval shape on magnetic activity, we perform a dedicated analysis of the ellipse parameters. The averaged $E_{\mathrm{m}}$ value and the five ellipse parameters are first derived for each $E_{\mathrm{m}}$ level. Subsequently we fit quadratic functions depending on $E_{\mathrm{m}}$ to each of the parameters for quantifying their dependence on activity:

$y=p_{2} \cdot E_{\mathrm{m}}^{2}+p_{1} \cdot E_{\mathrm{m}}+p_{0}$,

where $y$ represents any of the five ellipse parameters and $p_{0}, p_{1}$, and $p_{2}$ are coefficients determined by least-squares fitting. Figure 4 presents the obtained quadratic fits for the five parameters of the equatorward (left) and poleward (right) boundaries versus the merging electric field, $E_{\mathrm{m}}$. The black circles and red squares represent the Northern and Southern Hemisphere results, respectively. We can see that with increasing $E_{\mathrm{m}}$ both semi-axes of the ellipse expand to lower latitude. For large $E_{\mathrm{m}}$ values saturation effects appear for $\operatorname{semi}_{x}$ and semi $y$ of the equatorward boundary, while for the poleward boundary the saturation is limited to semi $y$ and $\operatorname{semi}_{x}$ is steadily increasing. The centers of the ellipses vary 

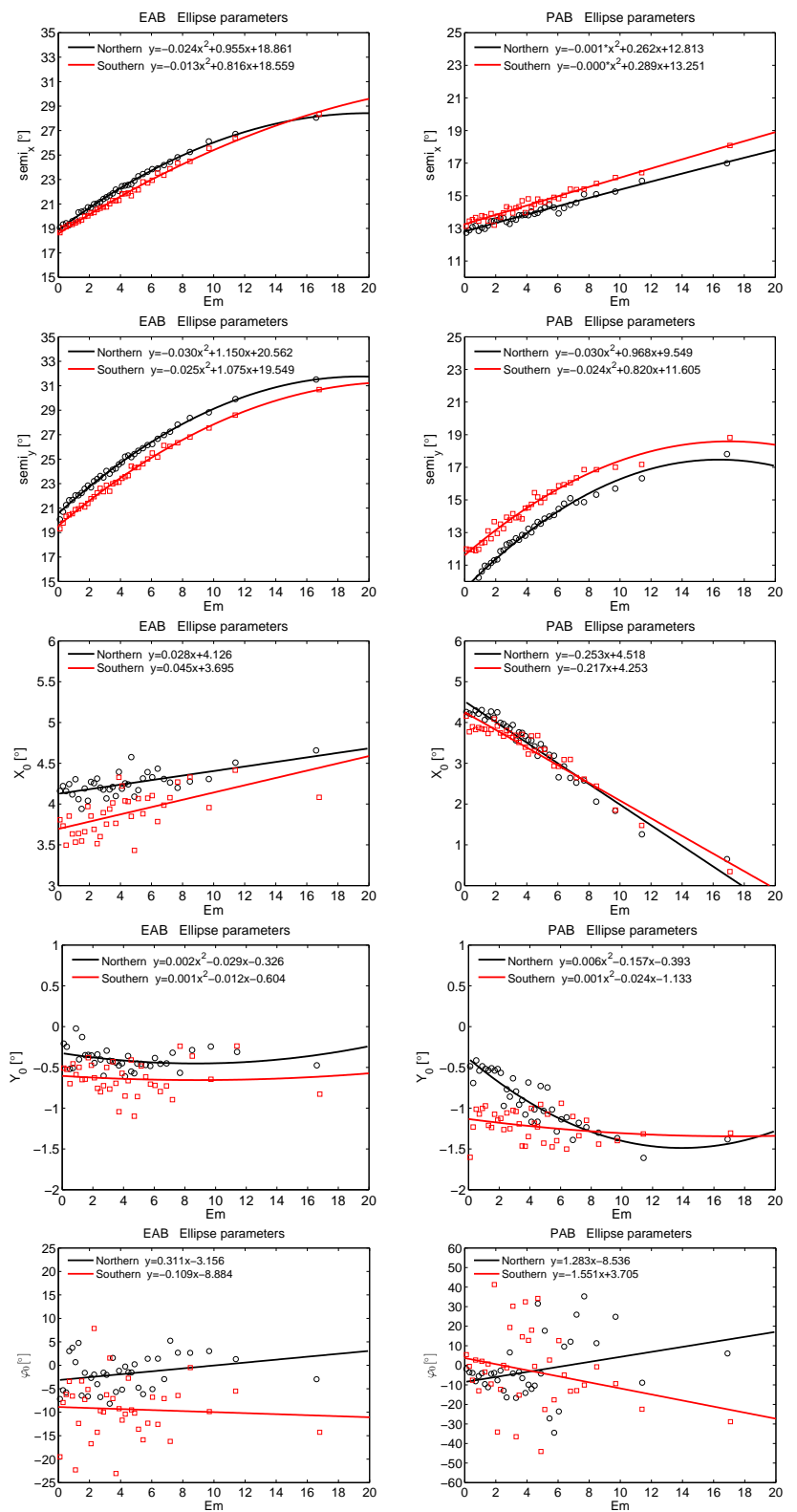

Figure 4. Quadratic fit of the ellipse parameter dependence on $E_{\mathrm{m}}$ for the equatorward (left) and poleward (right) auroral boundaries in both hemispheres.

only little. In the case of the equatorward boundary, $x_{0}$ indicates a shift away from the magnetic pole toward midnight with increasing $E_{\mathrm{m}}$; we observe an opposite reaction for the poleward boundary, as the center moves toward dayside. The component $y_{0}$ reflects only small displacements towards dusk for all levels of $E_{\mathrm{m}}$. Compared to the other four ellipse parameters, the rotation angle, $\varphi_{0}$, exhibits a rather scattered response to $E_{\mathrm{m}}$. For that reason, only linear dependences are derived. Since the boundaries have an almost circular shape, the angle is difficult to determine but the uncertainties of $\varphi_{0}$ have little effect. The coefficients for the
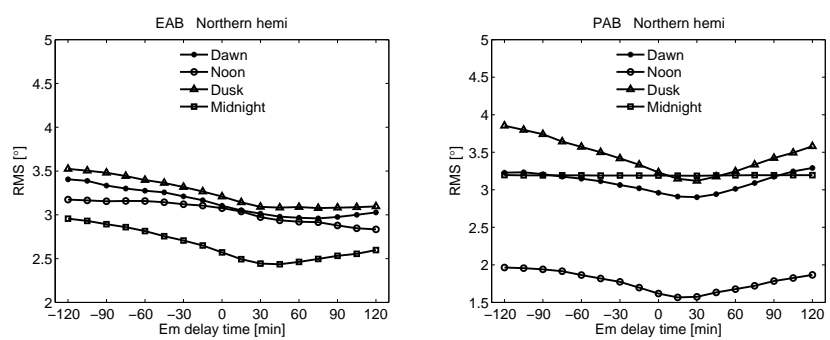

Figure 5. Determinations of optimal delay time for $E_{\mathrm{m}}$. The root mean square (RMS) values of the equatorward (left) and poleward (right) boundary residuals (the latitude differences between our $\mathrm{CH}$ Aurora-2014 model and CHAMP FAC detections) are shown for four different local-time sectors.

functional dependence of five parameters on $E_{\mathrm{m}}$ are listed in Tables 2 and 3. The derived functions allow for a continuous description of the auroral oval shape.

\subsection{The delay time}

Our aim is to make estimates of the auroral boundary locations based on the prevailing merging electric field, $E_{\mathrm{m}}$. It is expected that the boundaries exhibit a somewhat delayed response to variations of $E_{\mathrm{m}}$ acquired at the bow shock. Therefore, we try to find the appropriate delay times between $E_{\mathrm{m}}$ readings and the application of boundary prediction. To check our empirical model we have compared the individual boundary locations derived from FAC evaluations with those of the model predictions according to Eq. (4). For different delay times of $E_{\mathrm{m}}$ we determined the root mean square (RMS) values of the difference in latitude (defined as model prediction minus actual boundary detection). Such a test has been performed over a range of $\pm 2 \mathrm{~h}$ delay times at steps of $15 \mathrm{~min}$, separately for the dawn, noon, dusk, and midnight sectors. From Fig. 5 we can see that smallest RMS differences are found at 30 and 15 min delays for the equatorward and poleward boundaries, respectively. These delay times are used in all subsequent analyses. Figure 6 shows the distribution of boundary residuals between model and observations versus merging electric field after considering the optimal delay time for equatorward (left) and poleward (right) boundaries. Events from $2 \mathrm{~h}$ MLT bins have been selected around 06:00, 12:00, 18:00, and 00:00 MLT to represent dawn, noon, dusk, and midnight sectors. For all four MLT bins our empirical model shows rather consistent normal distributions of the residuals with respect to CHAMP boundary detections.

\subsection{Details of the auroral oval model}

For the construction of the new empirical model of the auroral oval we take advantage of the characteristics of the auroral boundaries derived from the CHAMP FAC signatures. Primary input for the model calculation is the value of $E_{\mathrm{m}}$. 
Table 2. Coefficients of the quadratic functions presenting the $E_{\mathrm{m}}$ dependence of the five ellipse parameters that describe the equatorward auroral boundary.

\begin{tabular}{|c|c|c|c|c|c|c|}
\hline Ellipse parameters $\left({ }^{\circ}\right)$ & \multicolumn{3}{|c|}{ Northern Hemisphere } & \multicolumn{3}{|c|}{ Southern Hemisphere } \\
\hline $\operatorname{semi}_{x}$ & $-2.3836 \times 10^{-2}$ & $9.5470 \times 10^{-1}$ & $1.8861 \mathrm{e} \times 10^{1}$ & $-1.3209 \times 10^{-2}$ & $8.1597 \times 10^{-1}$ & $1.8559 \mathrm{e} \times 10^{1}$ \\
\hline$x_{0}$ & 0 & $2.7827 \times 10^{-2}$ & $4.1263 \times 10^{0}$ & 0 & $4.4667 \times 10^{-2}$ & $3.6946 \times 10^{0}$ \\
\hline$y_{0}$ & $1.6569 \times 10^{-3}$ & $-2.8855 \times 10^{-2}$ & $-3.2637 \times 10^{-1}$ & $6.5985 \times 10^{-4}$ & $-1.1623 \times 10^{-2}$ & $-6.0436 \times 10^{-1}$ \\
\hline$\varphi_{0}$ & 0 & $3.1147 \times 10^{-1}$ & $-3.1555 \times 10^{0}$ & 0 & $-1.0934 \times 10^{-1}$ & $-8.8836 \times 10^{0}$ \\
\hline
\end{tabular}

Table 3. The same as Table 2, but for the poleward auroral boundary.

\begin{tabular}{|c|c|c|c|c|c|c|}
\hline \multirow[b]{2}{*}{ Ellipse parameters $\left({ }^{\circ}\right)$} & \multicolumn{3}{|c|}{ Northern Hemisphere } & \multicolumn{3}{|c|}{ Southern Hemisphere } \\
\hline & $p_{2}$ & $p_{1}$ & $p_{0}$ & $p_{2}$ & $p_{1}$ & $p_{0}$ \\
\hline $\operatorname{semi}_{x}$ & $-5.9729 \times 10^{-4}$ & $2.6173 \times 10^{-1}$ & $1.2813 \times 10^{1}$ & $-3.0559 \times 10^{-4}$ & $2.8870 \times 10^{-1}$ & $1.3251 \times 10^{1}$ \\
\hline $\operatorname{semi}_{y}$ & $-2.9556 \times 10^{-2}$ & $9.6759 \times 10^{-1}$ & $9.5486 \times 10^{0}$ & $-2.4073 \times 10^{-2}$ & $8.2006 \times 10^{-1}$ & $1.1605 \times 10^{1}$ \\
\hline$x_{0}$ & 0 & $-2.5310 \times 10^{-1}$ & $4.5175 \times 10^{0}$ & 0 & $-2.1674 \times 10^{-1}$ & $4.2526 \times 10^{0}$ \\
\hline$y_{0}$ & $5.6513 \times 10^{-3}$ & $-1.5732 \times 10^{-1}$ & $-3.9319 \times 10^{-1}$ & $7.0729 \times 10^{-4}$ & $-2.4479 \times 10^{-2}$ & $-1.1330 \times 10^{0}$ \\
\hline$\varphi_{0}$ & 0 & $1.2831 \times 10^{0}$ & $-8.5358 \times 10^{0}$ & 0 & $-1.5508 \times 10^{0}$ & $3.7050 \times 10^{0}$ \\
\hline
\end{tabular}

There is a four-step procedure intended for the prediction of the auroral boundaries.

1. Calculation of the control parameter $E_{\mathrm{m}}$, considering memory effect as defined by Eq. (2), and taking into account the delay times of 30 and 15 min for equatorward and poleward boundaries, respectively.

2. Using Eq. (8) and the parameters in Tables 2 and 3 to calculate the five ellipse parameters.

3. For a given magnetic local-time angle $\lambda$, the ellipse angle $\varphi$ in the first round has to be used as equal to $\lambda$ $(\varphi=\lambda)$ in Eqs. (3) and (4). In a second round, $\varphi$ is updated $(\varphi=\lambda+\Delta \lambda)$ for another application in Eqs. (3) and (4) according to Eqs. (5) and (6). The radius $r$ then gives the location of the auroral boundary in magnetic co-latitude.

4. It is recommended to adjust the model by actual boundary detections. This requires the following substeps.

a. Read the MLT and apex co-latitude of the CHAMP boundary crossing closest to the UT time of interest. Consider both crossings from ascending and descending arcs at the same pole, if available.

b. Calculate the predicted apex co-latitudes according to steps 1 to 3 for the CHAMP MLT and solar wind conditions.

c. Determine the mean difference in co-latitude, $\Delta r=$ $\left(r_{\mathrm{CH} 1}-r_{\bmod 1}+r_{\mathrm{CH} 2}-r_{\bmod 2}\right) / 2$, where the subscripts 1 and 2 denote ascending and descending crossings, respectively. d. Insert the adjustment of $\Delta r$ in Eq. (4)

$$
\begin{aligned}
& r^{\prime}= \\
& \sqrt{\left((r+\Delta r) \cdot \cos \left(\varphi+\varphi_{0}\right)+x_{0}\right)^{2}+\left((r+\Delta r) \cdot \sin \left(\varphi+\varphi_{0}\right)+y_{0}\right)^{2}}
\end{aligned}
$$

and repeat step 3 . The resulting position is in magnetic co-latitude (distance from the magnetic pole).

We term this new empirical mode $\mathrm{CH}$-Aurora-2014.

For times outside the CHAMP mission period (20002010) the model can be used with reduced accuracy. Results are then controlled entirely by the merging electric field, $E_{\mathrm{m}}$, and they are provided by the equations up to step 3 .

\section{Validation and discussion}

\subsection{Comparison with the Feldstein model}

The approach presented here for the first time uses fieldaligned current signatures for identifying the locations of auroral boundaries. For validating the predictions of the $\mathrm{CH}$ Aurora-2014 model, we have, as an initial step, taken the predictions of the Feldstein model (Holzworth and Meng, 1975) for comparison. The control parameter for the Feldstein model is the $Q$ index. Therefore, we first had to determine a suitable relation between our $E_{\mathrm{m}}$ and the $Q$ index. By using the $Q$ index and $E_{\mathrm{m}}$ data of the whole year 2005, we determined mean values of $E_{\mathrm{m}}$ for each $Q$ index. These mean values of $E_{\mathrm{m}}$ have been used as input for our $\mathrm{CH}$-Aurora2014 model. Figure 7 presents the predictions obtained of the equatorward (left) and poleward (right) auroral boundaries from the Feldstein model (black lines) and $\mathrm{CH}$-Aurora2014 (red lines) for three different $Q$ levels. In the case of the 

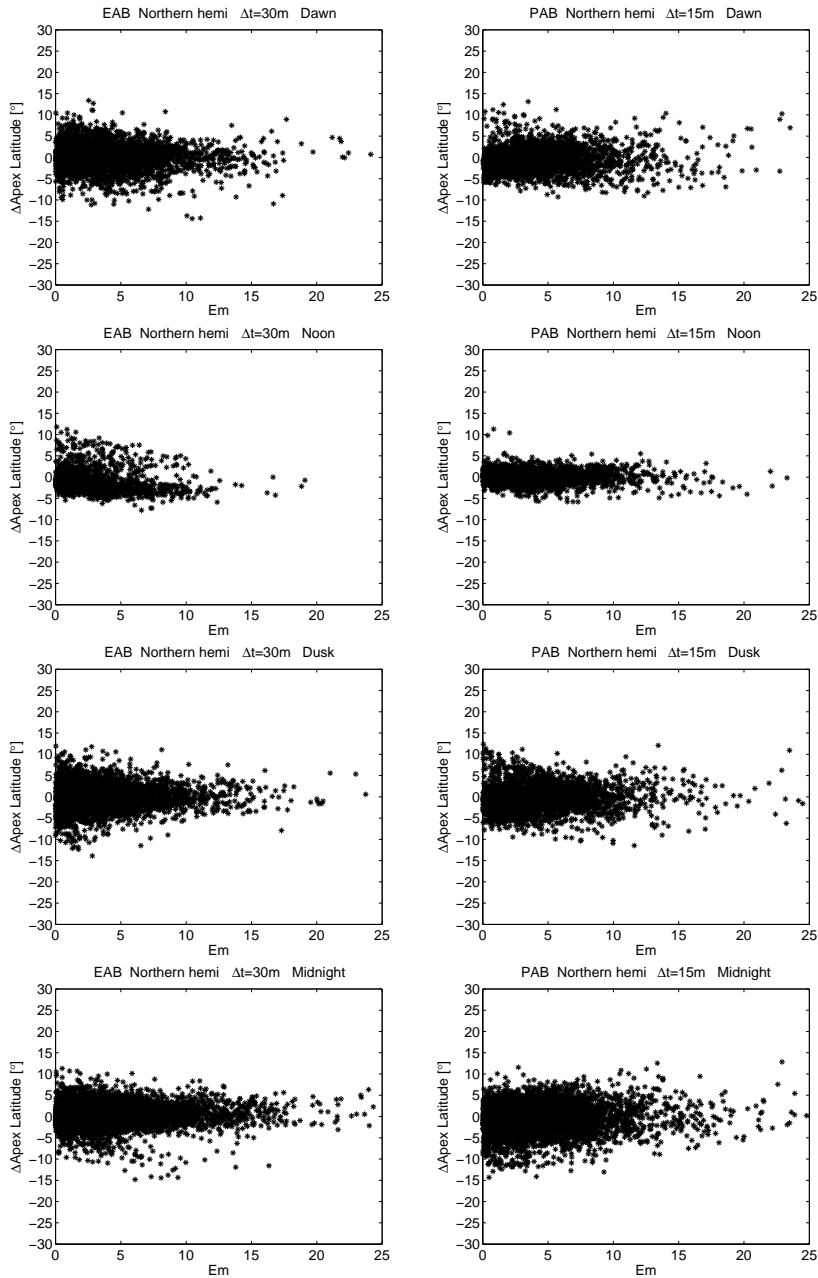

Figure 6. Distribution of the residuals (the latitude differences between our CH-Aurora-2014 model and CHAMP FAC detections) for equatorward (left) and poleward (right) boundaries. Positive values represent boundaries predicted too far poleward by $\mathrm{CH}$-Aurora2014.

equatorward boundary, the predictions from the two models are quite similar in the midnight sector but deviate clearly at other local times. $\mathrm{CH}$-Aurora-2014 predictions are about $2^{\circ}$ equatorward from the Feldstein model in most MLT sectors. The poleward auroral boundary is predicted by both models at almost the same latitude at midnight, while at the other local times the results differ considerably. In particular around noon, the Feldstein model shows peculiar latitude variations for high activity. This may be caused by uncertainties introduced by daylight.
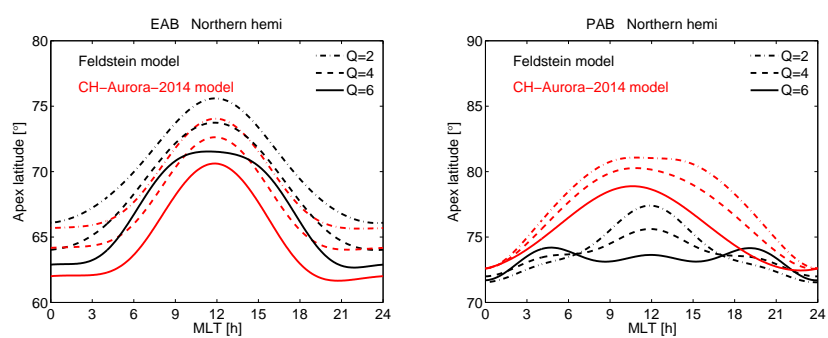

Figure 7. Comparison of the auroral equatorward (left) and poleward (right) boundaries between the Feldstein model (black lines) and our $\mathrm{CH}$-Aurora-2014 (red lines) for different magnetic activity levels; $Q=2,4,6$.

\subsection{Validation against the BAS auroral model}

A much more detailed test of the $\mathrm{CH}$-Aurora-2014 model was performed by a comparison with the BAS model. This more recent model is based on optical observations of the whole auroral oval by the IMAGE satellite. By means of an automatic detection scheme the auroral boundaries are detected (for details, see Longden et al., 2010). At intervals of 2 to $3 \mathrm{~min}$, the complete oval is recovered. The resulting database covering 2.5 years (May 2000-October 2002) of auroral boundaries in the Northern Hemisphere is freely available at the British Antarctic Survey server. The BAS model provides the auroral boundaries derived from three detectors of the far ultraviolet (FUV) imaging system instrument on board IMAGE; the boundaries derived from the Wideband Imaging Camera (WIC) have been used here for comparison.

In a one-to-one comparison we calculated the difference in latitude for every CHAMP pass when the BAS model was available. From the 2.5 year database of the BAS model, 2326 and 2295 common events were found for the equatorward and poleward boundaries, respectively. Generally we obtained a good match between the two independent observations. Figure 8 shows the distribution of the latitude differences. To first order, the difference distribution can be approximated by a Gauss curve well centered at zero. The distributions for the equatorward and poleward boundaries have similar shapes. For obtaining a more quantitative description of the differences between the models, two Gaussian functions are fitted to the distribution. The narrow (blue) curve represents the main body of observations, while the flat (red) curve represents the more serious outliers. Such a dual Gaussian distribution indicates the existence of two different type errors. The parameters of the two Gaussian curves are listed in Table 4. For both boundaries the width of the core distribution (sigma) is only $2^{\circ}$ in latitude. This holds for the main part of events. We find a small bias of about $0.5^{\circ}$ in latitude, indicating that CHAMP detections are more poleward. Differences larger than $5^{\circ}$ in latitudes (outside the blue curve) are considered as outliers and are not discussed further. Individual inspections showed that sometimes the BAS 
Table 4. Parameters of the Gaussian distributions fitted to the differences shown in Fig. 8.

\begin{tabular}{lrrrrr}
\hline & \multicolumn{2}{c}{$\mathrm{EAB}$} & & \multicolumn{2}{c}{ PAB } \\
\cline { 2 - 3 } \cline { 5 - 6 } \cline { 5 - 6 } & Inner curve & Outer curve & & Inner curve & Outer curve \\
\hline Amplitude $\left[{ }^{\circ}\right]$ & 16.28 & 3.29 & & 15.71 & 4.60 \\
Peak latitude $\left[{ }^{\circ}\right]$ & 0.33 & -0.21 & & 0.45 & 0.96 \\
Sigma $\left[{ }^{\circ}\right]$ & 2.07 & 4.86 & & 1.80 & 4.84 \\
\hline
\end{tabular}
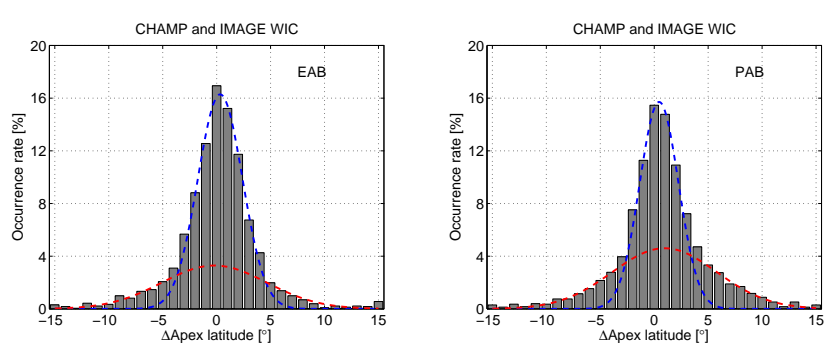

Figure 8. Distribution of the differences in latitude between the CHAMP FAC detections and IMAGE WIC auroral boundaries. The core part of the detections can be approximated well by a narrow Gaussian curve (blue). Obvious outliers follow a different distribution (red curve).

model is wrong and at other times our detection algorithm fails.

Besides the general distribution of the differences between the two models, we have also looked at the local-time distribution of the differences. For this analysis we used only events with differences of less than $\pm 5^{\circ}$. The top frame of Fig. 9 shows that during the early morning hours CHAMP detects the equatorward auroral boundary about $1^{\circ}$ further poleward than the boundary derived from IMAGE data. Conversely, the boundary deduced from optical observation appears $1^{\circ}$ in latitude further poleward during afternoon hours than that derived from CHAMP FAC activity. An explanation for this apparent discrepancy could be the polarity of region 2 (R2) FACs. In the morning sector R2 is associated with upward FAC. Therefore, auroras appear at the equatorward boundary, while in the afternoon little auroral activity is associated with downward R2 FACs. Based on these arguments we may suggest that the local-time-dependent bias is introduced by the BAS model. By contrast with the optical observations, enhanced small-scale FAC activity is present under both upward and downward FACs.

In the case of the poleward auroral boundary, there is little dependence of the differences on local time. Rather, we find a general poleward bias of the CHAMP detections. The average bias value of $0.5^{\circ}$ in latitude is also found in the Gaussian distribution (Fig. 8) and is listed in Table 4. We regard this bias value as a consequence of our definition of the thresholds in FAC activity that define auroral boundary. As described in Xiong et al. (2014), it is the mid-point of a significant increase of FAC intensity. Such systematic biases could be taken into account in a later model version.

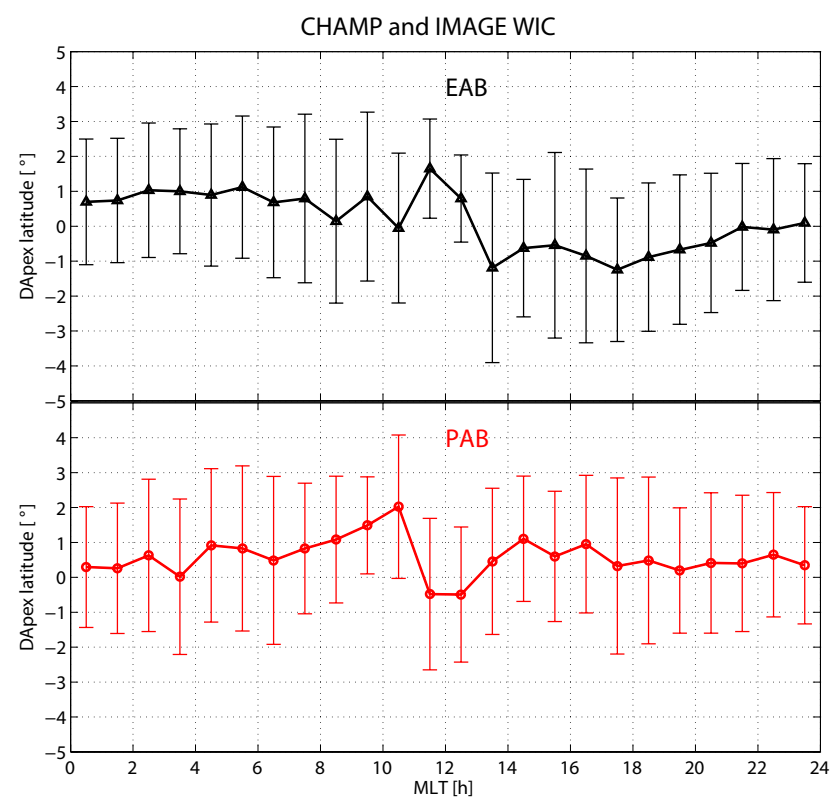

Figure 9. Hourly mean values and standard deviations of the localtime variation of latitude differences between auroral boundaries derived from CHAMP FAC and IMAGE WIC observations for equatorward (top) and poleward (bottom) auroral boundaries. Positive values indicate a poleward displacement of the CHAMP detections.

Around noon the differences of both boundaries vary significantly. We think this is caused by sunlight contamination that is disrupting to both detection methods.

\section{Summary and conclusion}

Here we have presented an empirical model of the auroral oval based on the enhanced small-scale FAC intensity in that region. High-resolution CHAMP magnetic field data of the years 2000-2010 from both hemispheres provide the required information. The basic shape of the oval is controlled by the dayside merging electric field and the size is adjusted to actual detections of the auroral boundaries by CHAMP. Some characteristics of the presented model $\mathrm{CH}-$ Aurora-2014 are:

1. The equatorward and poleward boundaries of the auroral oval can both be fitted well by ellipses for all levels of activity.

2. All five ellipse parameters show a dependence on the control parameter, the merging electric field, $E_{\mathrm{m}}$. The $E_{\mathrm{m}}$ dependence of the ellipse parameters can be fitted well by quadratic functions.

3. Best agreement between boundary detections and the prediction of its location is obtained when the merging electric field observed at the bow shock is delayed by 
$15 \mathrm{~min}$ for the poleward boundary and $30 \mathrm{~min}$ for the equatorward boundary. In addition, the memory effect of the ionosphere requires that the last $3 \mathrm{~h}$ of $E_{\mathrm{m}}$ should be taken into account.

4. A majority of the $\mathrm{CH}$-Aurora-2014 boundaries is in good agreement with the BAS model derived from IMAGE optical observations. Differences show a Gaussian distribution with a width of $\pm 2^{\circ}$ in latitude. Besides that, there are some obvious outliers which can be traced back to either of the two detection approaches.

5. There is a local-time-dependent difference of the equatorward boundary between the two models. CH-Aurora2014 finds the boundary $1^{\circ}$ polewards in the morning sector and $1^{\circ}$ in latitude equatorward during the afternoon sector of the BAS model. We think this is caused by the appearance of auroral forms in connection with upward FACs.

CHAMP detections of the auroral boundaries are available from August 2000 to September 2010. This is a sizeable data set for studying the dynamic of the oval for a wide range of ambient conditions. An extension of the series is intended based on Swarm data from 2014 onward. All required information and data needed for using CH-Aurora-2014 can be found at http://geomag.org/models/.

Acknowledgements. The BAS auroral boundary data were derived and provided by the British Antarctic Survey based on IMAGE satellite data. The CHAMP mission was sponsored by the Space Agency of the German Aerospace Center (DLR) through funds of the Federal Ministry of Economics and Technology. The work of Chao Xiong at GFZ is supported by the Alexander von Humboldt Foundation through a Research Fellowship for Postdoctoral Researchers.

The service charges for this open access publication have been covered by a Research Centre of the Helmholtz Association.

Topical Editor L. Blomberg thanks K. Lynch and one anonymous referee for their help in evaluating this paper.

\section{References}

Baker, D. N., Pulkkinen, T. I., Hesse, M., and McPherron, R. L.: A quantitative assessment of energy storage and release in the Earth's magnetotail, J. Geophys. Res., 102, 7159-7168, doi:10.1029/96JA03961, 1997.

Boakes, P. D., Milan, S. E., Abel, G. A., Freeman, M. P., Chisham, G., Hubert, B., and Sotirelis, T.: On the use of IMAGE FUV for estimating the latitude of the open/closed magnetic field line boundary in the ionosphere, Ann. Geophys., 26, 2759-2769, doi:10.5194/angeo-26-2759-2008, 2008.

Feldstein, Y. I.: Some problems concerning the morphology of auroras and magnetic disturbances at high latitudes, Geomagn. Aeron., 3, 183-195, 1963.

Feldstein, Y. I. and Starkov, G. V.: The auroral oval and the boundary of closed field lines of geomagnetic field, Planet. Space. Sci., 15, 501-508, 1970.

Frey, H. U.: Localized aurora beyond the auroral oval, Rev. Geophys., 45, RG1003, doi:10.1029/2005RG000174, 2007.

Hardy, D. A., Gussenhoven, M. S., and Holeman, E.: A statistical model of auroral electron precipitation, J. Geophys. Res., 90, 4229-4248, 1985.

Hardy, D. A., Holeman, E. G., Burke, W. J., Gentile, L. C., and Bounar, K. H.: Probability distributions of electron precipitation at high magnetic latitudes, J. Geophys. Res., 113, A06305, doi:10.1029/2007JA012746, 2008.

Holzworth, R. and Meng, C.: Mathematical representation of the auroral oval, Geophys. Res. Lett., 2, 337-380, 1975.

Kan, J. R. and Lee, L. C.: Energy coupling function and solar windmagnetosphere dynamo, Geophys. Res. Lett., 6, 577-580, 1979.

Kauristie, K., Weygand, J., Pulkkinen, T. I., Murphree, J. S., and Newell, P. T.: Size of the auroral oval: UV ovals and precipitation boundaries compared, J. Geophys. Res., 104, 2321-2331, 1999.

Khorosheva, O. V.: Spatial-temporal distribution of aurorae, in Aurorae, no. 16, 1-84, Academy of Sciences, Moscow, 1967 (in Russian).

Liu, R., Lühr, H., Doornbos, E., and Ma, S.-Y.: Thermospheric mass density variations during geomagnetic storms and a prediction model based on the merging electric field, Ann. Geophys., 28, 1633-1645, doi:10.5194/angeo-28-1633-2010, 2010.

Longden, N., Chisham, G., Freeman, M. P., Abel, G. A., and Sotirelis, T.: Estimating the location of the open-closed magnetic field line boundary from auroral images, Ann. Geophys., 28, 1659-1678, doi:10.5194/angeo-28-1659-2010, 2010.

Newell, P. T., Sotirelis, T., Liou, K., Meng, C.-I., and Rich, F. J.: A nearly universal solar wind-magnetosphere coupling function inferred from 10 magnetospheric state variables, J. Geophys. Res., 112, A01206, doi:10.1029/2006JA012015, 2007.

Werner, S. and Prölss, G. W.: The position of the ionospheric trough as a function of local time and magnetic activity, Adv. Space Res., 20, 1717-1722, 1997.

Xiong, C., Lühr, H., Wang, H., and Johnsen, M. G.: Determining the boundaries of the auroral oval from CHAMP fieldaligned current signatures - Part 1, Ann. Geophys., 32, 609-622, doi:10.5194/angeo-32-609-2014, 2014. 